\title{
Prognostic factors of depression and depressive symptoms after hip fracture surgery: systematic review
}

\author{
R. Milton-Cole ${ }^{1 *}$, S. Ayis ${ }^{1}$, K. Lambe ${ }^{1}$, M. D. L. O'Connell ${ }^{1}$, C. Sackley ${ }^{1,2}$ and K. J. Sheehan ${ }^{1}$
}

\begin{abstract}
Background: Patients with hip fracture and depression are less likely to recover functional ability. This review sought to identify prognostic factors of depression or depressive symptoms up to 1 year after hip fracture surgery in adults. This review also sought to describe proposed underlying mechanisms for their association with depression or depressive symptoms.

Methods: We searched for published (MEDLINE, Embase, PsychInfo, CINAHL and Web of Science Core Collection) and unpublished (OpenGrey, Greynet, BASE, conference proceedings) studies. We did not impose any date, geographical, or language limitations. Screening (Covidence), extraction (Checklist for critical Appraisal and data extraction for systematic Reviews of prediction Modelling Studies, adapted for use with prognostic factors studies Checklist), and quality appraisal (Quality in Prognosis Studies tool) were completed in duplicate. Results were summarised narratively.

Results: In total, 37 prognostic factors were identified from 12 studies included in this review. The quality of the underlying evidence was poor, with all studies at high risk of bias in at least one domain. Most factors did not have a proposed mechanism for the association. Where factors were investigated by more than one study, the evidence was often conflicting.

Conclusion: Due to conflicting and low quality of available evidence it is not possible to make clinical recommendations based on factors prognostic of depression or depressive symptoms after hip fracture. Further high-quality research investigating prognostic factors is warranted to inform future intervention and/or stratified approaches to care after hip fracture.
\end{abstract}

Trial registration: Prospero registration: CRD42019138690.

Keywords: Hip fracture, Depression, Prognostic factors, Predictors

\section{Introduction}

Hip fractures are among the most common orthopaedic injuries affecting 66,313 older adults in the United Kingdom in 2018 [1]. These fractures can negatively affect patients' health-related quality of life as they often lead to losses in

\footnotetext{
* Correspondence: rhian.1.milton-cole@kcl.ac.uk

'Department of Population Health Sciences, King's College London, School of Population Health and Environmental Sciences, Guy's Campus, London SE1 1UL, UK

Full list of author information is available at the end of the article
}

mobility and independence [2], a need for ongoing care, limitations in activities of daily living (ADLs) [3] and ensuing death [4]. To mitigate these risks, prompt surgery and a subsequent period of rehabilitation is the definitive management approach for most hip fractures [5].

Psychiatric illness, including depression and depressive symptoms, is common in the population of older adults with hip fracture [6]. The reported prevalence of depression among patients with hip fracture is between 9 and $47 \%$, depending on the country, population, duration of

C C The Author(s). 2021 Open Access This article is licensed under a Creative Commons Attribution 4.0 International License, which permits use, sharing, adaptation, distribution and reproduction in any medium or format, as long as you give appropriate credit to the original author(s) and the source, provide a link to the Creative Commons licence, and indicate if changes were made. The images or other third party material in this article are included in the article's Creative Commons licence, unless indicated otherwise in a credit line to the material. If material is not included in the article's Creative Commons licence and your intended use is not permitted by statutory regulation or exceeds the permitted use, you will need to obtain permission directly from the copyright holder. To view a copy of this licence, visit http://creativecommons.org/licenses/by/4.0/. The Creative Commons Public Domain Dedication waiver (http://creativecommons.org/publicdomain/zero/1.0/) applies to the data made available in this article, unless otherwise stated in a credit line to the data. 
depressive symptoms, the method used to assess depression and the type of hip fracture [7, 8]. Patients with depression or depressive symptoms are less likely to recover functioning (as measured by function, e.g., balance or walking speed, and activities, e.g., activities of daily living) after hip fracture compared to those without depression [9]. The risk of developing depression or depressive symptoms is highest before discharge and in the 12 months following the event [10]. Indeed, Maharlouei and colleagues reported depression as a major contributing prognostic factor for recovery after hip fracture [11] and those with consistently high levels of depressive symptoms following hip fracture were at a considerably increased risk of not returning to their baseline physical function [12].

Several studies have identified prognostic factors for depression or depressive symptoms after hip fracture [13-15]. These prognostic factor studies investigate which characteristics are associated with changes in depressive symptoms or the occurrence of new-onset depression [16]. The purpose of such studies is to gain a better understanding of the disease process and to define risk groups based on outcome prognosis [17]. This would enable the development of new interventions or quality improvement initiatives targeting modifiable prognostic factors and/or stratified approaches to care for non-modifiable prognostic factors [16]. In addition, while a given factor may not be modifiable, the proposed underlying mechanisms for its association with the outcome may be modifiable [18], further informing interventions and quality improvement initiatives.

To date, there has been no attempt to synthesise the evidence on prognostic factors of depression or depressive symptoms after hip fracture or to assess the underlying mechanisms for the association between these factors and depression or depressive symptoms. This is important as an understanding of the extent and nature of prognostic factors of depression or depressive symptoms could help to inform future approaches to optimise recovery after hip fracture. Therefore, this review aims to identify prognostic factors of depression or depressive symptoms up to 1 year after hip fracture surgery in adult patients. The secondary aim is to summarise the proposed underlying mechanisms for their association with depression or depressive symptoms.

\section{Methods}

The protocol for this systematic review is registered on the International Prospective Register of Systematic Reviews (PROSPERO: CRD42019138690).

\section{Eligibility criteria}

This review included studies of prognostic factors for depression or depressive symptoms in adults over the age of 18 who have undergone surgery for a nonpathological hip fracture. We adopted an inclusive approach to eligibility with exclusions limited to children (those below the age of 18 years), those treated for a pathological fracture, and those treated conservatively. No geographical, language or date limits were applied.

\section{Search strategy}

Databases were searched to identify relevant published (MEDLINE, Embase, PsychInfo, CINAHL, Web of Science Core Collection) and unpublished (OpenGrey, Greynet and Bielefeld Academic Search Engine (BASE) and conference proceedings) studies from inception to 9th November 2020. Reference lists of included studies were reviewed for any further relevant studies. The search strategy was developed using previously published search terms and synonyms identified in the search strategies of published Cochrane reviews conducted on hip fractures and depression [19-22], terminology used in NICE guidelines, through the MeSH database and Ovid MEDLINE subject heading function. The search strategy also included the published recommended search strategy for identifying prognostic factor studies (Supplementary File 1) [23].

\section{Study selection}

References were exported into Covidence for deduplication and screening [24]. Two reviewers screened the titles and abstracts independently and then carried out full-text screening against the eligibility criteria. Conflicts were resolved by consensus or by a third author if consensus could not be reached.

\section{Data extraction}

Three authors extracted data for all included studies independently using the modified Checklist for critical Appraisal and data extraction for systematic Reviews of prediction Modelling Studies, adapted for use with prognostic factors studies (CHARMS-PF checklist) [25]. Information extracted included the authors' names, publication year, study dates, setting and design, timepoints definitions, outcomes, prognostic factors information, sample size, analysis methods, and results data. Data on the proposed underlying mechanisms for reported associations were also extracted. Any disagreements during this stage were resolved by consensus. If any information was missing or incomplete, an attempt was made to contact the study authors to retrieve the missing data.

\section{Quality appraisal}

Three authors assessed the methodological quality of all included studies independently using the refined QUality In Prognosis Studies (QUIPS) appraisal tool. QUIPS is a 
six-domain checklist used to assess risk of bias in prognostic factor studies (at the study level) [26]. The domains are study participation, study attrition, prognostic factor measurement, outcome measurement, study confounding and statistical analysis and reporting. Grading of each domain consisted of three options: high, moderate, or low risk of bias. Disagreements in risk of bias judgements were resolved by consensus.

\section{Analysis}

We evaluated the data extraction table for homogeneity between studies. There was substantial heterogeneity in study design, prognostic factors investigated, and their methods of measurements, study timepoints, methods of analysis and reporting of results. Therefore, conducting a meta-analysis was not possible. We reported the results in a narrative synthesis using text, figures, and tables [27]. We organised factors according to whether they related to the patient or care structures and processes, and their proposed underlying mechanisms.

\section{Results}

\section{Study selection}

We identified 3402 studies from five databases, 462 of which were duplicates. During title/abstract and full-text screening, 2915 studies were excluded. On full text review we excluded studies by population where control groups who had not suffered a hip fracture were used and the results were limited to comparisons between those with and without hip fracture and where the sample included any patient who sustained fall-related injuries to any limb $(n=4)$, study design as the level of depression in patients with cognitive impairments was investigated rather than the prognostic factors of depression or depressive symptoms and the study was of crosssectional design $(n=2)$, outcome; where outcomes included the effect of ageing on immunity, the prognosis of mental disorders, the impact and cause of depression after hip fracture, functional recovery and the prognosis of outcomes in those with depression after hip fracture $(n=5)$, and timing when follow up times were up to 2 years after discharge and 3 years after hospitalisation $(n=2)$. Therefore, 12 studies were included in this review. Study selection is summarised in Fig. 1.

\section{Study characteristics and measures of depression}

Characteristics of each study are summarised in Table 1 below. This review included 12 studies consisting of 2642 patients. The sample size ranged from 23 [32] to 570 [14], with a median sample size of 146 . The mean age of patients ranged from 76.2 [32] - 81.8 [13] years. Depression or depressive symptoms were measured using the Depression Anxiety Stress Scale in one study [28], the Montgomery- Åsberg Depression Rating Scale in three studies [15, 30, 35], Structured Clinical Interview for DSM-IV disorders (SCID-IV) in two studies $[15,32]$, the Geriatric Depression Scale - Short Form (GDS-SF) in one study [29], the Geriatric Depression Scale in three studies [31, 34, 35], the Geriatric Depression Scale-Chinese version in two studies [10,33], the Hamilton Depression Rating Scale (Ham-D) in two studies [13, 32], Primary Care Evaluation of Mental Disorders in one study [13] and the Hospital Anxiety and Depression Scale (HADS) in two studies [14, 35].

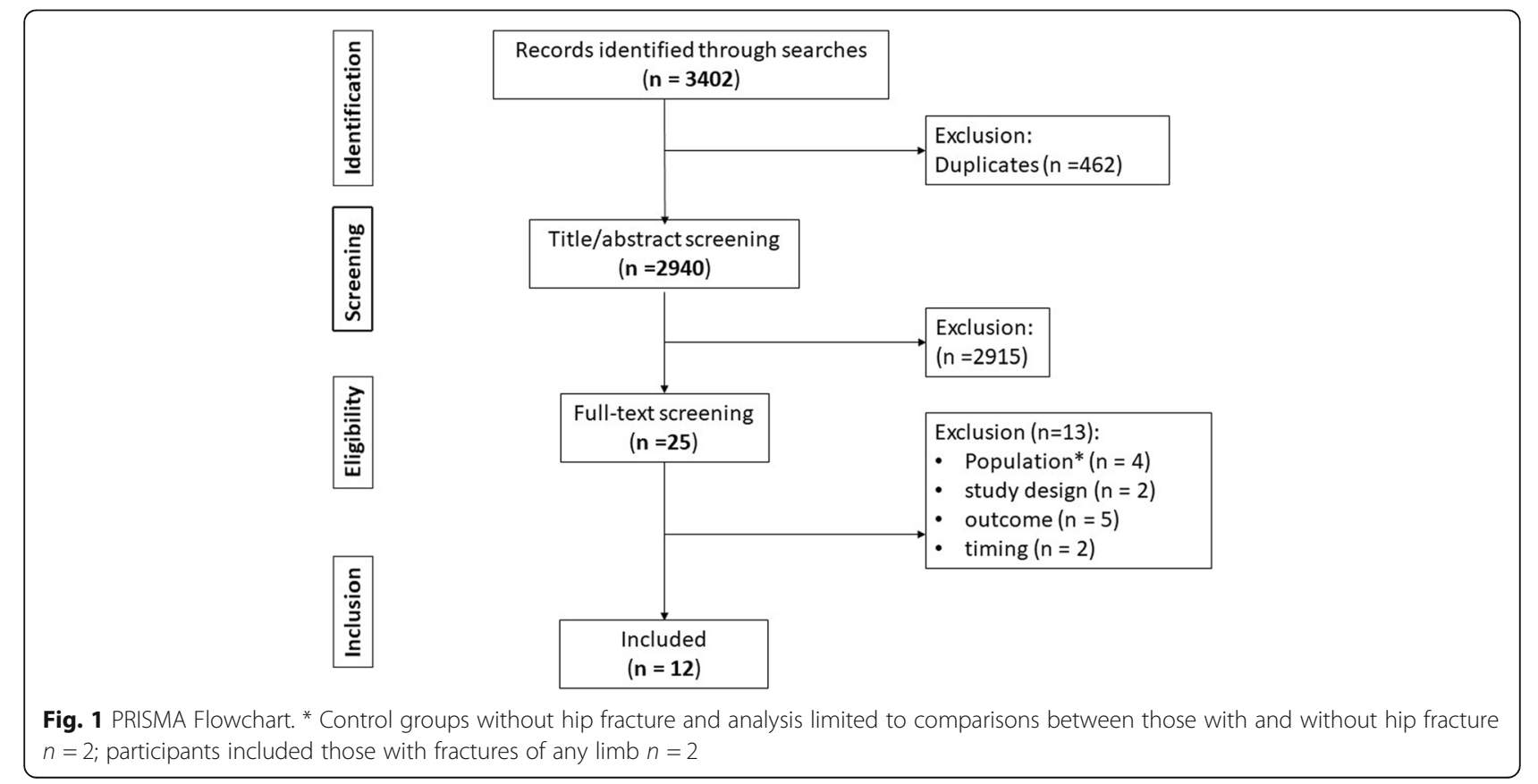




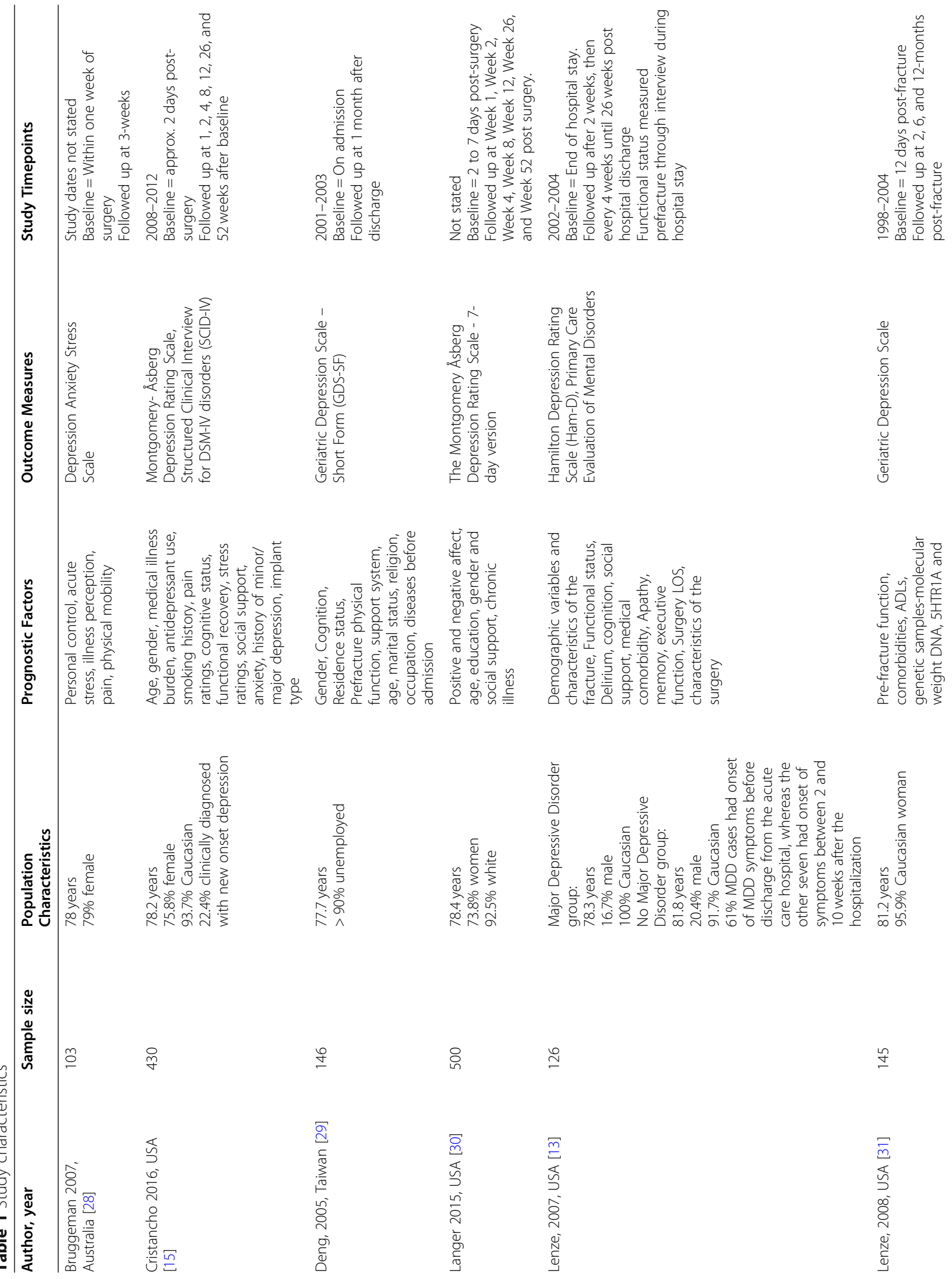




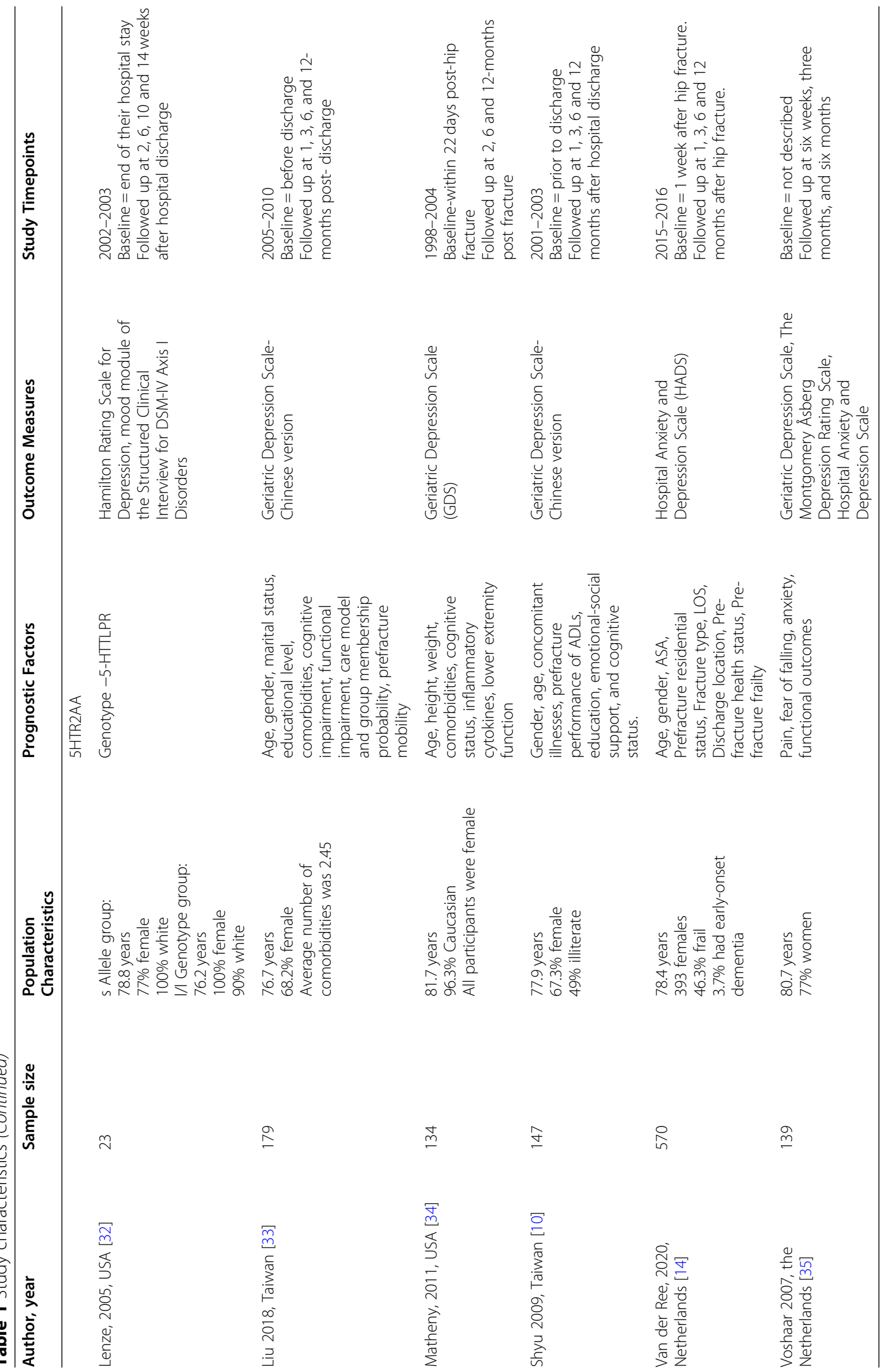


Baseline timepoints (where reported) ranged from on admission [29] to prior to discharge [10, 13, 32, 33] or 22 days post-hip fracture [34]. Follow up time points ranged from 1-week post-surgery [30] to 12 months after hip fracture/surgery $[10,14,30,31,33,34]$.

\section{Risk of bias in studies}

The quality of the studies included in this review was variable, with 10 of 12 studies at high risk of bias in at least one domain and no study at low risk of bias across all domains (Table 2). Three out of the 12 studies were at moderate risk of bias for study participation [28-30], while the other nine studies were at low risk of bias $[10,13-15,31-$ 35]. Five studies were at high risk of bias for study attrition $[15,28-30,34]$, four studies were judged to be at moderate risk [14, 31, 33, 35], and the others deemed to be at low risk of bias [10, 13, 32]. One study [29] was judged to be at high risk of bias for prognostic factor measurement, six out of the 12 studies were at moderate risk of bias [10, $15,28,33-35]$, and the remaining five studies were at low risk [13, 14, 30-32]. One study was at high risk of bias for outcome measurement [29], with all other studies deemed at low risk. Study confounding had the highest risk of bias overall, with seven studies being high risk [10, 14, 15, 29, $30,32,35]$, two studies were at moderate risk of bias [28, $33]$ and three studies at low risk [13, 31, 34]. Statistical analysis and reporting were at high risk of bias in five studies [10, 29, 32-34], moderate risk in five studies [13, $15,30,31,35]$ and two studies were judged to be at low risk of bias [14, 28]. Detailed rationale for each risk of bias assignment is presented in Supplementary File 2.

\section{Prognostic factors}

A total of 37 prognostic factors were investigated across the 12 studies included in this review (Table 3). Most studies did not identify a primary prognostic factor of interest (rather reporting on multiple factors from one model). Lenze [32] was the only study to identify a primary prognostic factor reporting a positive association between Genotype -5-HTTLPR and depression or depressive symptoms after hip fracture. Factors explored by more than one study included age $[13-15,30]$, gender $[13-15,29,30]$, cognitive status [13, 15, 29], comorbidities [13, 15, 31, 33], fracture type [13, 14], anxiety $[15,35]$, pain $[15,28,35]$, residence status $[14,29]$, social support $[13,15,29,30]$, stress $[15,28]$, activities of daily living $[15,29,31,33]$, mobility $[14,15,28,35]$ and function $[13,35]$. Four authors were contacted for additional information however this additional information was not available $(n=2)$, or the authors did not respond to our request $(n=2)$.

\section{Patient-related factors}

Twelve studies explored 35 factors relating to patient characteristics. Four factors were accompanied by a proposed underlying mechanism for their reported association.

Apathy [13], anxiety [15, 35], discharge location [14], inflammatory cytokines [34], personal control beliefs [28], current smoking status [15], negative affect [30], American Society of Anaesthesiologists (ASA) [14], history of depression [15], antidepressant use [15], and pre-fracture frailty [14] were positively associated with depression or depressive symptoms after hip fracture. Delirium [13], hopelessness [28], fear of falling [35], chronic illness [30], executive function [13], memory [13], pre-fracture health status [14], education [30] and fracture type [13, 14] were not associated with depression or depressive symptoms after hip fracture. Four studies reported no association between age and depression or depressive symptoms after hip fracture $[13-15,30]$. There was no positive association reported between residence status and depression or depressive symptoms after hip fracture investigated by two studies

Table 2 Results of Quality Appraisal using the Quality In Prognosis Studies (QUIPS) Tool Summary

\begin{tabular}{|c|c|c|c|c|c|c|}
\hline Author, year & $\begin{array}{l}\text { Study } \\
\text { participation }\end{array}$ & $\begin{array}{l}\text { Study } \\
\text { attrition }\end{array}$ & $\begin{array}{l}\text { Prognostic factor } \\
\text { measurement }\end{array}$ & $\begin{array}{l}\text { Outcome } \\
\text { measurement }\end{array}$ & $\begin{array}{l}\text { Study } \\
\text { confounding }\end{array}$ & $\begin{array}{l}\text { Statistical analysis/ } \\
\text { reporting }\end{array}$ \\
\hline Bruggeman 2007, Australia [28] & moderate & high & moderate & low & moderate & Low \\
\hline Cristancho 2016, USA [15] & low & high & moderate & low & high & moderate \\
\hline Deng, 2005, Taiwan [29] & low & high & high & high & high & high \\
\hline Langer 2015, USA [30] & moderate & high & low & low & high & moderate \\
\hline Lenze, 2007, USA [13] & low & low & low & low & moderate & moderate \\
\hline Lenze, 2008, USA [31] & low & moderate & low & low & low & moderate \\
\hline Lenze, 2005, USA [32] & low & low & low & low & high & high \\
\hline Liu 2018, Taiwan [33] & low & moderate & moderate & low & moderate & high \\
\hline Matheny, 2011, USA [34] & low & high & moderate & low & low & high \\
\hline Shyu 2009, Taiwan [10] & low & low & moderate & low & high & high \\
\hline Van der Ree, 2020, Netherlands [14] & low & moderate & low & low & high & low \\
\hline Voshaar 2007, the Netherlands [35] & low & moderate & moderate & low & high & moderate \\
\hline
\end{tabular}




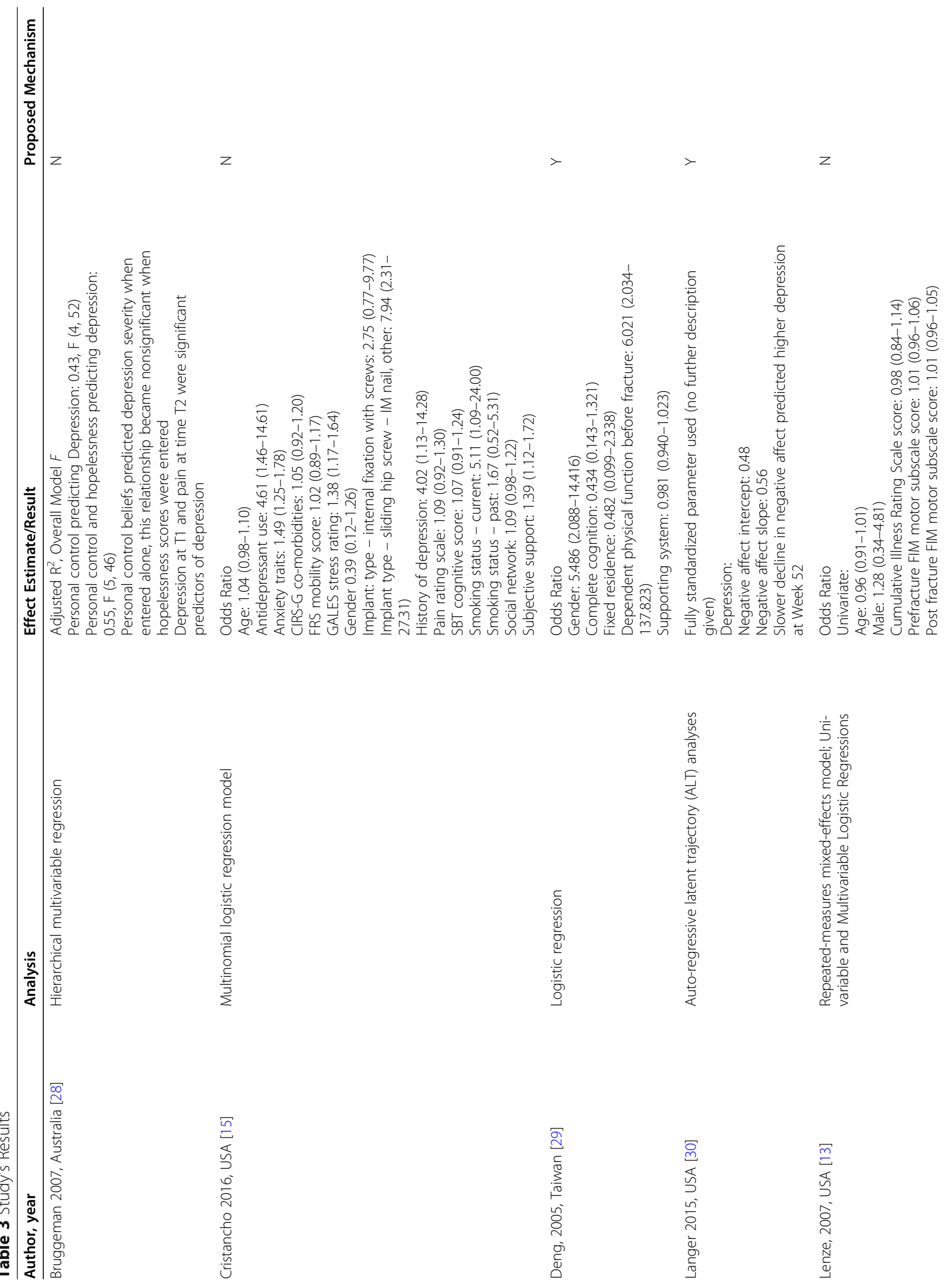




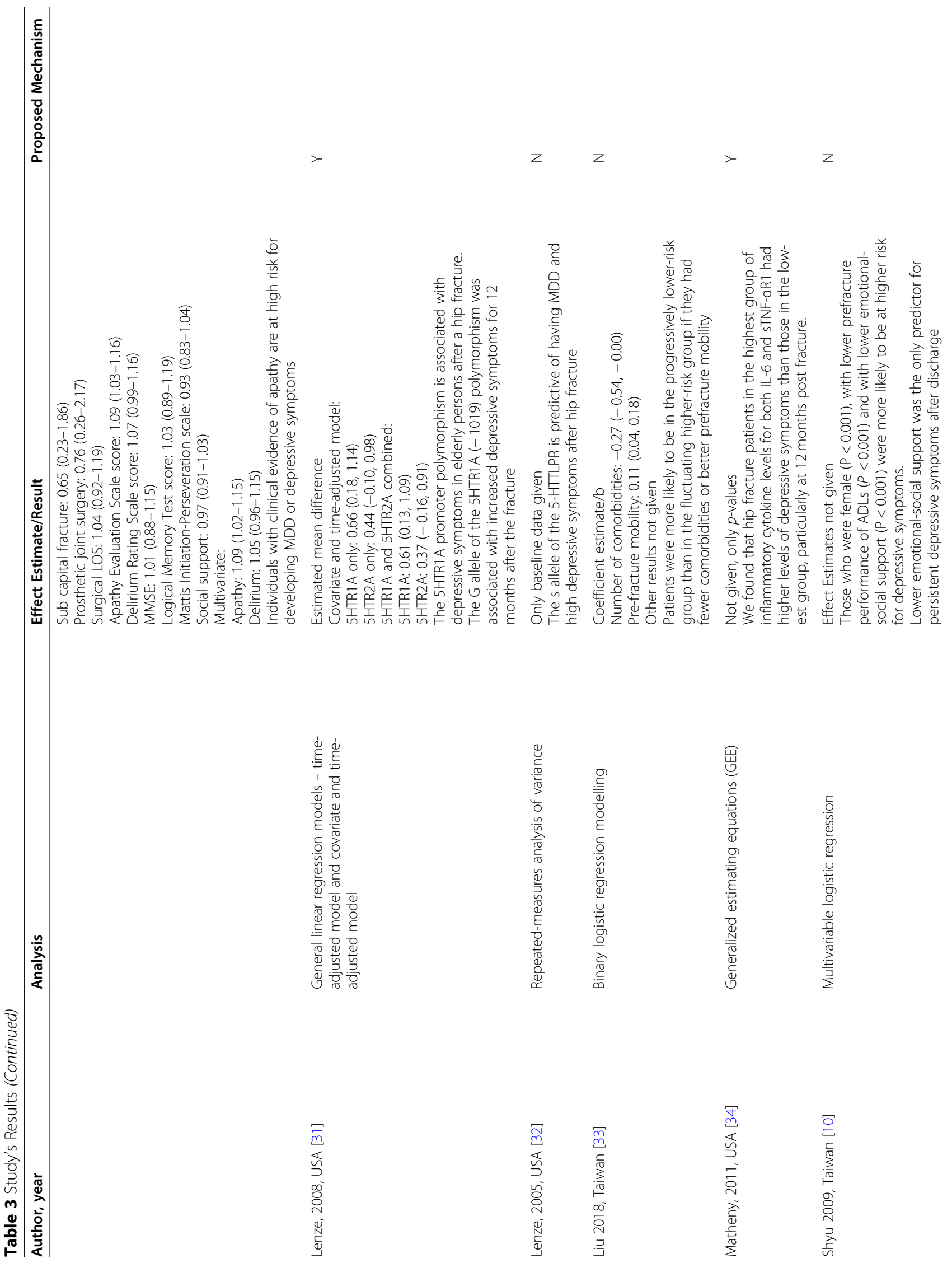


$\begin{array}{ll}\text { Milton-Cole et al. BMC Geriatrics } & \text { (2021) 21:537 }\end{array}$

Page 9 of 15

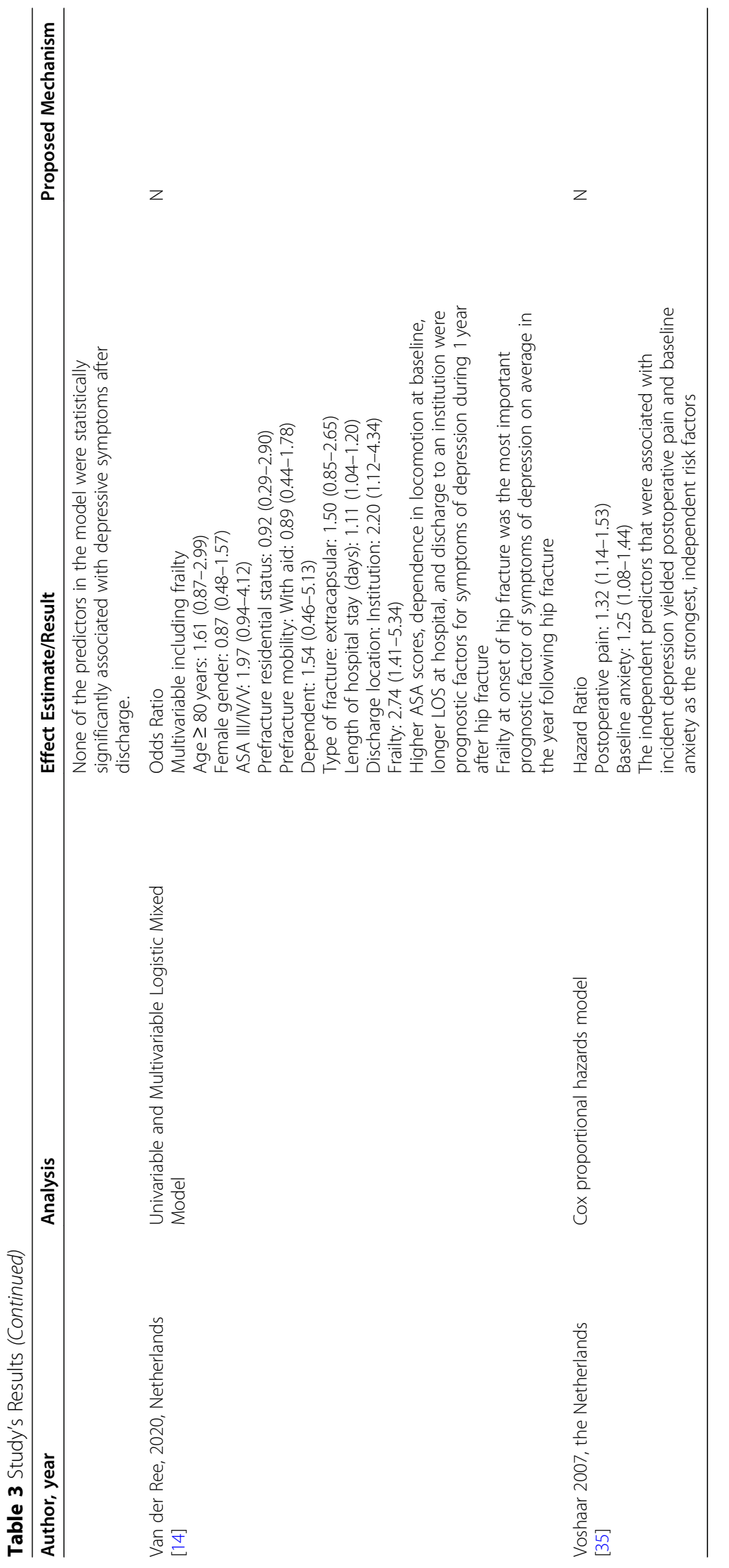


$[14,29]$. There was conflicting evidence for an association between pain and depression or depressive symptoms after hip fracture, two studies reporting a positive association $[28,35]$ and one study reporting no association [15]. Similarly, two studies reported conflicting evidence for a positive association between stress and depression or depressive symptoms after hip fracture $[15,28]$. Three studies reported no association between lack of social support and depressive symptoms after hip fracture [13, 29, 30].

In contrast, one study reported low social support was associated with depression or depressive symptoms in hip fracture patients [15]. One study reported an association between lower performances of activities of daily living and depression or depressive symptoms after hip fracture (measured using the Chinese version of the Barthel Index) [33]. Another study reported an association between lower performances of activities of daily living and instrumental activities of daily living (measured using the Chinese version of the Barthel Index and the Instrumental activities of daily living scale (IADLs)) with depression or depressive symptoms after hip fracture [29], while two studies reported no association [15, 31]. These studies measured activities of daily living and instrumental activities of daily living using Basic activities of daily living (BADLs) and IADLs scales [15] and the Lower extremity Physical activities of daily living and IADLs scales [31] respectively. One study reported an association [14] and three reported no association [15, $28,35]$ between reduced mobility and depression or depressive symptoms after hip fracture. Two studies reported no association between lower function (measured by Functional Independence Measure, gait test (the time and number of steps taken in a 4-m walk) and the functional reach test) and depression or depressive symptoms after hip fracture [13, 35].

There were further inconsistent findings for the association between gender, comorbidities and pre-fracture residence and depression or depressive symptoms after hip fracture. Four studies reported no association between gender and depression or depressive symptoms after hip fracture $[13-15,30]$. In contrast, Deng reported women were five times more likely to have depression or depressive symptoms than men [29]. Four studies investigated the association between comorbidities and depressive symptoms [13, 15, 31, 33]. One study reported an association suggesting patients with more comorbidities were more likely to be in the higher risk group for depression or depressive symptoms than those with less comorbidities [33]. Three studies reported no association between a lower cognitive status and depressive symptoms $[13,15$, 29]. Two studies reported an association between the presence of genotypes 5HTR1A and 5-HTTLPR [31, 32] and no association between 5HTR2A [31] and depression or depressive symptoms after hip fracture.
Underlying mechanisms Most studies did not propose an underlying mechanism for the association between their prognostic factor/s of interest and depression or depressive symptoms after hip fracture. Lenze [31] reported 5HTR1A was predictive of depressive symptoms due to the interaction between genetics and socialenvironmental stressors. Deng [29] proposed the role shift from caregiver to care-receiver resulting in feelings of conflict between their physical ability and social expectations, as a potential mechanism for their reported association between gender and depression or depressive symptoms. Matheny [34] proposed an underlying mechanism that the increased cytokines may indicate a chronic sickness syndrome or due to the transient stimulation of these cytokines by physical and psychological stressors for their reported association between social support and depression or depressive symptoms. A proposed mechanism of the association between social support and depression or depressive symptoms after hip fracture is an extensive social network may have a protective role in times of distress [30].

\section{Process/structure-related factors}

Three studies explored factors related to care processes or structures [13-15]. Two studies investigated the association between a longer length of stay $[13,14]$ and depression or depressive symptoms after hip fracture. Lenze [13] reported no association between length of stay and depression or depressive symptoms after hip fracture. In contrast, van de Ree [14] reported a longer length of hospital stay was associated with depression or depressive symptoms in the year after hip fracture. The type of surgery was not associated with depression or depressive symptoms in one study (39\% of participants received prosthetic joint surgery, other types of surgery are not described) [13]. In contrast, in the study by Cristancho [15], implant type was associated with depression or depressive symptoms whereby patients who had a sliding hip screw and intramedullary nail were more likely to develop depression or depressive symptoms compared to patients who had an internal fixation with screws [15]. No study proposed an underlying mechanism for the association between these factors and depression or depressive symptoms after hip fracture. Figure 2 shows all prognostic factors investigated and whether an association was reported with depression or depressive symptoms.

\section{Discussion}

We identified 37 prognostic factors of depression or depressive symptoms after hip fracture surgery across 12 studies. Most studies investigated patient factors, with only a few related to care process or structure factors. Few studies proposed an underlying mechanism for the 


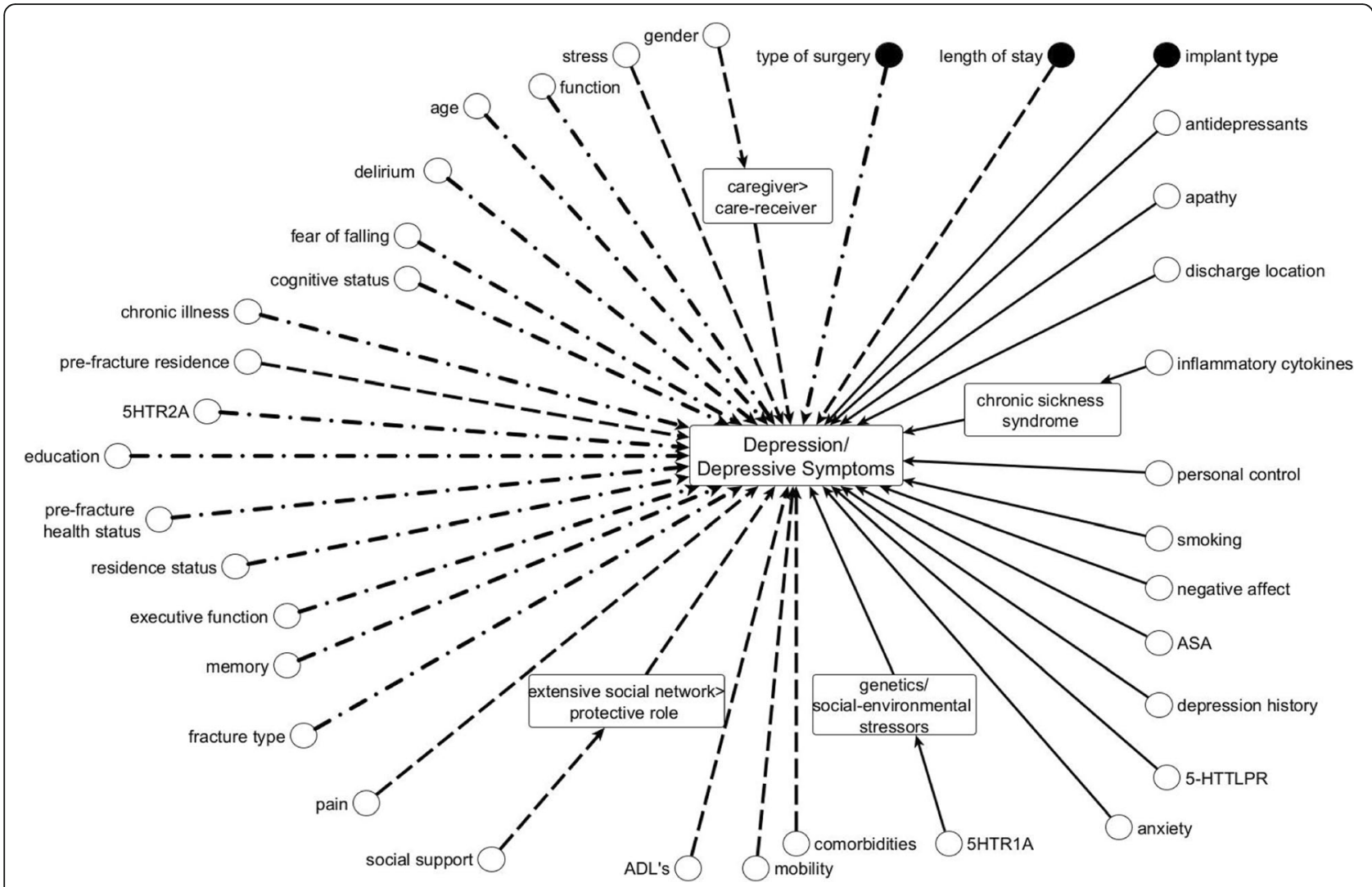

Fig. 2 Prognostic Factors of Depression or Depressive Symptoms identified in this review. Nodes represent prognostic factors. Dashed arrows indicate conflicting evidence for the presence of an association. Straight arrows indicate a reported association. Unequal dashed arrows indicate no reported association. The rounded edge square boxes indicate reported underlying mechanisms. Black nodes denote structure/process factors

reported association. For factors assessed by more than one study, there was often conflicting evidence which may be attributed to the heterogeneity of the studies. Synthesis of the study's results was challenging due to heterogeneity in study design, methods of assessments for the prognostic factors and depression or depressive symptoms, time points evaluated, and the prognostic factors investigated. Therefore, identifying which prognostic factors are the strongest predictors of depression or depressive symptoms after hip fracture surgery was not possible. This was further compounded by the lack of studies explicitly defining their primary prognostic factor. This approach introduces the phenomenon known as the "Table 2 fallacy" [36], where the effect estimates of secondary prognostic factors are inappropriately interpreted alongside the estimates of the primary prognostic factor. This may lead to an overestimation of the association between these secondary prognostic factors and outcomes as models are likely insufficient to control for confounding of secondary factors associated with the outcome. Further, the prognostic factors taken into consideration are often highly correlated, and one factor may work as a proxy for another. Analysing such factors together may render a factor unimportant, yet in another study where no correlated factors were considered, the same factor may be important.

Only three studies were deemed to be at low risk of bias for attrition. An additional concern related to attrition was a failure to report the extent of missing data across several studies [13, 28, 32, 33]. Missing data can lead to invalid conclusions due to a reduction in the study's statistical power, representativeness of the study sample, and bias in the estimations made [37]. For the current review, the rate of attrition likely increased with the severity of depressive symptoms and therefore data is likely not missing at random [38]. This presents challenges for analysis as common missing data techniques (e.g., imputation) are not appropriate for data not missing at random but the estimate of the effect may be biased due to the missingness [39]. This limits the generalizability of the prognostic factors identified to those with more severe depression/depressive symptoms.

The current review highlights a dearth of evidence investigating structures or processes of care. A longer length of stay was identified as a prognostic factor for depressive symptoms in the first year after hip fracture. It has previously been shown that depression can increase a patient's length of hospital stay after hip fracture surgery [40]. This 
potentially indicates a bi-directional relationship in which experiencing depressive symptoms increases a patient's length of stay as well as a longer length of stay being a predictor of depressive symptoms after hip fracture surgery. However, the association was disputed by Lenze [13], and therefore this relationship warrants further study. Similarly, inconsistency in the evidence for an association between surgery type and depression or depressive symptoms was reported [13, 15]. All studies failed to propose an underlying mechanism for putative associations between structures, processes, and depression or depressive symptoms. Failure to identify a plausible underlying mechanism could result in observing a statistical association in the absence of causation.

Most studies identified patient factors associated with depression or depressive symptoms after hip fracture. For example, one study reported the inflammatory cytokines interleukin-6 (IL-6) and tumour necrosis factoralpha (sTNF- $\alpha$ R1) were associated with depressive symptoms 1 year after hip fracture [34]. These inflammatory markers are also associated with adverse outcomes postoperatively, such as complications [41]. These unfavourable outcomes may be what leads to depressive symptoms in hip fracture patients postoperatively. IL-6, a pro-inflammatory marker, is involved in the disease progression of osteoarthritis [42]. While no therapies targeting IL-6 pathway inhibitors in individuals with osteoarthritis have been developed, the antibody tocilizumab is an effective treatment in certain conditions when IL-6 levels are increased [42]. Future research into therapies blocking the pro-inflammatory cytokines IL-6 and sTNF- $\alpha \mathrm{R} 1$ in hip fracture patients may provide an intervention which influences the development of depression or depressive symptoms after hip fracture. There were inconsistencies between studies on whether pain was associated with depression or depressive symptoms after hip fracture. This difference may be due to the variations in end points. The two studies which found an association followed-up at 3 weeks [28] and up to 6 months [35] compared to the study that did not find an association that followed up to a year after baseline [15]. Acute pain has previously been associated with depression this may explain the differences in findings [43]. We noted that some prognostic factors were modifiable and are therefore amenable to change through intervention which in turn leads to improvements in patient's quality of care.

Most factors we identified were non-modifiable factors. Understanding such factors allow healthcare professionals to stratify patients according to their risk of depression or depressive symptoms after hip fracture [44]. For example, the $\mathrm{G}$ allele of the 5HTR1A polymorphism, a serotonin receptor, is associated with depressive symptoms 1 year after hip fracture in the older population. Due to the connection between genetics and socio-environmental stressors, this association was not examined in-depth by the authors. Future research focusing on this may aid in establishing whether specific genotypes are predictive of depression or depressive symptoms after hip fracture enabling targeted intervention for individuals with these genotypes. Pre fracture frailty is also associated with depression after hip fracture. Previous literature demonstrates that the presence of either frailty or depression increases the prevalence and incidence of the other [45]. Therefore, stratifying individuals after hip fracture surgery by frailty status allows those most at risk of developing depression to receive specialised management.

Several predictors identified by this review are themselves depressive symptoms namely personal control, hopelessness, negative affect, apathy, and anxiety. Here it was noted that anxiety $[15,35]$ and negative affect [30] were predictors of more severe depressive episodes as measured by the Montgomery-Åsberg Depression Rating Scale, and apathy [13] was predictive of minor depressive disorders. One study reported a positive association for personal control and no association for hopelessness with depressive symptoms as measured by the Depression, Anxiety and Stress Scale [28]. This is somewhat surprising given both personal control and hopelessness are considered related and symptoms of depression [46, 47]. Indeed, a lack of personal control often leads to feelings of hopelessness and subsequent clinical depression $[48,49]$. The surprising result may be due to the poor methodological quality of the study which was at moderate to high risk of bias across five of six QUIPS domains. Alternatively, the findings may suggest certain depressive symptoms may be related to the severity of specific depressive subtypes. For example, the hopelessness theory of depression hypothesizes the negative causal attribution made by individuals in response to adverse life events creates a sense of hopelessness, which can lead to a distinct cognitively mediated subtype of depression, hopelessness depression [47]. It is possible the Depression, Anxiety and Stress Scale may not be sensitive to this subtype.

Previous literature shows that anxiety and apathy often coexist in complex relationships but are distinct entities $[50,51]$. In the general population, the coexistence of general anxiety with depressive symptoms is significant, as is the confounding effect the presence of one has on the other [50]. There is also an overlap between the phenomena of apathy and depression or depressive symptoms [51]. However, the extent to which outcome measures for depression or depressive symptoms are sensitive to this has been discussed in the literature [52] Previous studies have shown measures of these factors are highly correlated with depression in multiple 
populations [52]. Therefore, it may not be possible to state whether true anxiety and apathy are prognostic factors of depression or depressive symptoms in this review.

\section{Strengths and limitations}

In this review, screening of published and unpublished literature using broad eligibility criteria (including no language restrictions), data extraction, and quality appraisal were completed in duplicate, reducing the risk of bias. We did not search for registered ongoing studies, which may have led to underestimating the extent of prognostic factors. We employed the Quality In Prognosis Studies (QUIPS) tool for the quality assessments. The tool recommends reviewers give an overall risk of bias judgement to studies by deciding the most important domain a priori; the assigned judgement for these specific domains is then used to determine the overall study risk of bias [53]. However, this may potentially lead to bias as the selection of the most important domains is subjective as there is no literature on which domains are the most significant [54]. We, therefore, did not provide a judgement on the overall risk of bias. We were not able to perform quantitative synthesis due to insufficient data; this limited our ability to provide a scientifically rigorous summary of the results [55]. We also adopted broad eligibility criteria and did not set a minimum sample size. This led to the inclusion of studies of varying methodological quality, including those with a small sample size. These studies have poor precision in their estimates due to the small sample, which further limits our ability to draw conclusions from the results.

We employed a broad definition of depression and/or depressive symptoms in our eligibility criteria to identify all potentially relevant literature. However, it is possible factors prognostic of depressive symptoms may/may not be prognostic of a clinical diagnosis of depression. This may have led to an overestimation or an underestimation of the extent of relevant prognostic factors. Additionally, two studies $[15,30]$ appear to be from the same population (unable to confirm with authors) which may have led to an overestimation of the number of studies reporting no association between age or gender and depression/depressive symptoms after hip fracture.

We did not perform a quantitative synthesis due to insufficient data. This decision was made following review of data extraction. We identified 14 prognostic factors that were reported by more than one study. For each of these factors, no study explicitly identified a primary prognostic factor of interest and associated appropriate potential confounders, rather interpreting multiple effect estimates from one regression model. As previously specified, this approach is not recommended [36] due to risk of bias known as the Table 2 Fallacy [36].
Unfortunately, for seven prognostic factors, the multivariable analysis was not accompanied by univariable analyses for each prognostic factor limiting the potential for metaanalysis from univariable results. Where univariable analysis was reported, there was heterogeneity in factor measurement e.g., length of stay was measured by surgical [13] and by total length of stay [14] or outcome measurement e.g., comorbidities as a prognostic factor of depressive symptoms [31] and of major depressive disorders [15] or in effect estimates e.g., linear regression [33] and logistic regression [29] to evaluate the prognostic association between activities of daily living and depressive symptoms, and insufficient crude data provided to generate new comparable estimates. Therefore, we did not deem the evidence sufficiently homogenous to warrant exploration with quantitative synthesis. This limited our ability to provide a more rigorous summary of the results [55].

This review focused on those with hip fracture, therefore the results may not be generalisable to the older adult population. We excluded studies with a non-hip fracture control group where the study's results were limited to comparisons between those with and without hip fracture. We took this approach so the results could be directly applied to the hip fracture population however, this may have led to an underestimation of the number of predictors. Further, we did not include data from outcome measures whose sub-components may include questions related to depressive symptoms e.g., EQ5D. This may have led to an underestimation of the extent to which prognostic factors of depression or depressive symptoms after hip fracture have been explored in the available literature.

\section{Conclusions}

The current review identified 37 prognostic factors of depression or depressive symptoms after hip fracture surgery across 12 studies. Where factors were investigated by more than one study, there was often conflicting evidence and no proposed mechanism for the reported associations. It is therefore not possible to make any clinical recommendations based on the available evidence. Further high-quality research investigating prognostic factors is warranted to inform future intervention and/or stratified approaches to care after hip fracture.

Abbreviations

PRISMA: Preferred Reporting Items for Systematic reviews and Meta-Analyses; QUIPS: QUality In Prognosis Studies; ADLs: Activities of Daily Living

\section{Supplementary Information}

The online version contains supplementary material available at https://doi. org/10.1186/s12877-021-02514-1.

Additional file 1: Appendix 1. Search strategies.

Additional file 2. 


\section{Acknowledgements}

We would like to thank Ms. Gergana Manolova who supported quality appraisal of the studies included in this review.

\section{Authors' contributions}

RMC, KJS, SA, MDLOC, and CS contributed to the conception and design of the study. In addition, RMC, KJS, and KL contributed to the acquisition and the analysis of data. All authors contributed to the interpretation of the analysis. RMC drafted the manuscript. All authors critically revised the manuscript. All authors approved the final version for submission.

\section{Funding}

This work was supported by a UKRI Future Leaders Fellowship [Grant Ref: $\mathrm{MR} / \mathrm{S} 032819 / 1]$

\section{Availability of data and materials}

Data sharing does not apply to this article as no datasets were generated or analysed during the current study.

\section{Declarations}

\section{Ethics approval and consent to participate}

Not applicable - No ethical approval was required as this was a systematic review that analysed published data.

\section{Consent for publication}

Not applicable.

\section{Competing interests}

KS received funding from the NIHR Research for Patient Benefit, Chartered Society of Physiotherapy Charitable Trust and UKRI Future Leaders Fellowship for hip fracture health services research and is on the Editorial board of BMC Geriatrics. RMC's PhD studentship is funded as a host contribution to KS UKR Future Leaders Fellowship. SA is funded by the NIHR Biomedical Research Centre based at Guy's and St Thomas' NHS Foundation Trust, King's College London. MOC is on the Editorial board of BMC Geriatrics. CS receives funding from the NIHR HTA and Global Health streams. None of the authors have published or submitted any related papers from the same study. This work forms a part of a PhD programme of work by RMC. This studentship is funded as a host contribution to a UKRI Future Leaders Fellowship (Grant Ref: MR/S032819/1).

\section{Author details}

'Department of Population Health Sciences, King's College London, School of Population Health and Environmental Sciences, Guy's Campus, London SE1 1UL, UK. ${ }^{2}$ Faculty of Medicine and Health Sciences, University of Nottingham, Nottingham, UK.

Received: 14 May 2021 Accepted: 16 September 2021

Published online: 10 October 2021

\section{References}

1. Physicians RCo. National hip Fracture Database annual report 2019. London: RCP; 2019.

2. Castelli A, Daidone $S$, Jacobs R, Kasteridis P, Street AD. The determinants of costs and length of stay for hip fracture patients. PLoS One. 2015;10(7): e0133545. https://doi.org/10.1371/journal.pone.0133545.

3. Dyer SM, Crotty M, Fairhall N, Magaziner J, Beaupre LA, Cameron ID, et al. A critical review of the long-term disability outcomes following hip fracture. BMC Geriatr. 2016;16(1):158. https://doi.org/10.1186/s12877-016-0332-0.

4. Haentjens P, Magaziner J, Colon-Emeric CS, Vanderschueren D, Milisen K, Velkeniers $B$, et al. Meta-analysis: excess mortality after hip fracture among older women and men. Ann Intern Med. 2010;152(6):380-90. https://doi. org/10.7326/0003-4819-152-6-201003160-00008.

5. NICE. The management of hip fracture in adults. London: National Clinical Guideline Centre; 2017

6. Nightingale S, Holmes J, Mason J, House A. Psychiatric illness and mortality after hip fracture. Lancet. 2001;357(9264):1264-5. https://doi.org/10.1016/ S0140-6736(00)04421-4.

7. Holmes JD, House AO. Psychiatric illness in hip fracture. Age Ageing. 2000; 29(6):537-46. https://doi.org/10.1093/ageing/29.6.537.
8. Heidari ME, Naghibi Irvani SS, Dalvand P, Khadem M, Eskandari F, Torabi F, et al. Prevalence of depression in older people with hip fracture: a systematic review and meta-analysis. Int J Orthop Trauma Nurs. 2021;40: 100813. https://doi.org/10.1016/j.ijotn.2020.100813.

9. Phillips AC, Upton J, Duggal NA, Carroll D, Lord JM. Depression following hip fracture is associated with increased physical frailty in older adults: the role of the cortisol: dehydroepiandrosterone sulphate ratio. BMC Geriatr. 2013;13(1):60. https://doi.org/10.1186/1471-2318-13-60.

10. Shyu Yl, Cheng HS, Teng HC, Chen MC, Wu CC, Tsai WC. Older people with hip fracture: depression in the postoperative first year. J Adv Nurs. 2009; 65(12):2514-22. https://doi.org/10.1111/j.1365-2648.2009.05125.x.

11. Maharlouei $N$, Jafarzadeh $F$, Lankarani KB. Factors affecting recovery during the first 6 months after hip fracture, using the decision tree model. Arch Osteoporos. 2019;14(1):61. https://doi.org/10.1007/s11657-019-0611-4.

12. Mossey JM, Knott $K$, Craik R. The effects of persistent depressive symptoms on hip fracture recovery. J Gerontol. 1990;45(5):M163-8. https://doi.org/10.1 093/geronj/45.5.M163.

13. Lenze EJ, Munin MC, Skidmore ER, Dew MA, Rogers JC, Whyte EM, et al. Onset of depression in elderly persons after hip fracture: implications for prevention and early intervention of late-life depression. J Am Geriatr Soc. 2007:55(1):81-6. https://doi.org/10.1111/j.1532-5415.2006.01017.x.

14. van de Ree CLP, de Munter L, Biesbroeck BHH, Kruithof N, Gosens T, de Jongh MAC. The prevalence and prognostic factors of psychological distress in older patients with a hip fracture: a longitudinal cohort study. Injury. 2020;51(11):2668-75. https://doi.org/10.1016/j.injury.2020.07.049.

15. Cristancho P, Lenze EJ, Avidan MS, Rawson KS. Trajectories of depressive symptoms after hip fracture. Psychol Med. 2016;46(7):1413-25. https://doi. org/10.1017/S0033291715002974.

16. Riley $R$, van der Windt $D$, Croft $P$, Moons KGM. Prognosis research in healthcare: concepts, methods, and impact. Oxford: Oxford University Press; 2019. p. 354.

17. Altman DG, Lyman GH. Methodological challenges in the evaluation of prognostic factors in breast cancer. Breast Cancer Res Treat. 1998;52(1-3): 289-303. https://doi.org/10.1023/A:1006193704132.

18. Sheehan KJ, Williamson L, Alexander J, Filliter C, Sobolev B, Guy P, et al. Prognostic factors of functional outcome after hip fracture surgery: a systematic review. Age Ageing. 2018:47(5):661-70. https://doi.org/10.1093/a geing/afy057.

19. Crotty M, Unroe K, Cameron ID, Miller M, Ramirez G, Couzner L. Rehabilitation interventions for improving physical and psychosocial functioning after hip fracture in older people. Cochrane Database Syst Rev. 2010;(1):CD007624. https://doi.org/10.1002/14651858.CD007624.pub3.

20. Farooqi V, van den Berg ME, Cameron ID, Crotty M. Anabolic steroids for rehabilitation after hip fracture in older people. Cochrane Database Syst Rev. 2014;(10):CD008887. https://doi.org/10.1002/14651858.CD008887.pub2.

21. Ijaz S, Davies P, Williams CJ, Kessler D, Lewis G, Wiles N. Psychological therapies for treatment-resistant depression in adults. Cochrane Database Syst Rev. 2018; 5(8):CD010558. https://doi.org/10.1002/14651858.CD010558.pub2.

22. Smith CA, Armour M, Lee MS, Wang LQ, Hay PJ. Acupuncture for depression. Cochrane Database Syst Rev. 2018;3:CD004046.

23. Geersing GJ, Bouwmeester W, Zuithoff P, Spijker R, Leeflang M, Moons KG. Search filters for finding prognostic and diagnostic prediction studies in Medline to enhance systematic reviews. PLoS One. 2012;7(2):e32844. https:// doi.org/10.1371/journal.pone.0032844

24. Harrison H, Griffin SJ, Kuhn I, Usher-Smith JA. Software tools to support title and abstract screening for systematic reviews in healthcare: an evaluation. BMC Med Res Methodol. 2020;20(1):7. https://doi.org/10.1186/s12874-0200897-3.

25. Riley RD, Moons KGM, Snell KIE, Ensor J, Hooft L, Altman DG, et al. A guide to systematic review and meta-analysis of prognostic factor studies. BMJ. 2019;364:k4597. https://doi.org/10.1136/bmj.k4597.

26. Hayden JA, Cote P, Bombardier C. Evaluation of the quality of prognosis studies in systematic reviews. Ann Intern Med. 2006;144(6):427-37. https:// doi.org/10.7326/0003-4819-144-6-200603210-00010.

27. Siddaway AP, Wood AM, Hedges LV. How to do a systematic review: a best practice guide for conducting and reporting narrative reviews, Metaanalyses, and Meta-syntheses. Annu Rev Psychol. 2019;70(1):747-70. https:// doi.org/10.1146/annurev-psych-010418-102803.

28. Bruggemann L, Nixon RD, Cavenett T. Predicting acute anxiety and depression following hip fracture. J Behav Med. 2007:30(2):97-105. https:// doi.org/10.1007/s10865-006-9088-x. 
29. Deng H, Shyu YL, Chen M, Tasi Y, Lin H. Factors related to depression in elderly patients receiving hospitalization care for hip fractures. J Evid Based Nurs. 2005;1(4):253-62.

30. Langer JK, Weisman JS, Rodebaugh TL, Binder EF, Lenze EJ. Short-term affective recovery from hip fracture prospectively predicts depression and physical functioning. Health Psychol. 2015;34(1):30-9. https://doi.org/10.103 7/hea0000111.

31. Lenze EJ, Shardell M, Ferrell RE, Orwig D, Yu-Yahiro J, Hawkes W, et al. Association of serotonin-1A and $2 \mathrm{~A}$ receptor promoter polymorphisms with depressive symptoms and functional recovery in elderly persons after hip fracture. J Affect Disord. 2008;111(1):61-6. https://doi.org/10.1016/j.jad.2008. 02.005 .

32. Lenze EJ, Munin MC, Ferrell RE, Pollock BG, Skidmore E, Lotrich F, et al. Association of the serotonin transporter gene-linked polymorphic region (5HTTLPR) genotype with depression in elderly persons after hip fracture. Am J Geriatr Psychiatry. 2005;13(5):428-32. https://doi.org/10.1097/00019442-2 00505000-00014.

33. Liu HY, Yang CT, Tseng MY, Chen CY, Wu CC, Cheng HS, et al. Trajectories in postoperative recovery of elderly hip-fracture patients at risk for depression: a follow-up study. Rehabil Psychol. 2018;63(3):438-46. https://doi.org/10.103 7/rep0000130.

34. Matheny ME, Miller RR, Shardell MD, Hawkes WG, Lenze EJ, Magaziner J, et al. Inflammatory cytokine levels and depressive symptoms in older women in the year after hip fracture: findings from the Baltimore hip studies. J Am Geriatr Soc. 2011;59(12):2249-55. https://doi.org/10.1111/j.1 532-5415.2011.03727.x

35. Voshaar RC, Banerjee S, Horan M, Baldwin R, Pendleton N, Proctor R, et al. Predictors of incident depression after hip fracture surgery. Am J Geriatr Psychiatry. 2007;15(9):807-14. https://doi.org/10.1097/JGP.0b013e318098610c

36. Westreich D, Greenland S. The table 2 fallacy: presenting and interpreting confounder and modifier coefficients. Am J Epidemiol. 2013;177(4):292-8. https://doi.org/10.1093/aje/kws412.

37. Kang $H$. The prevention and handling of the missing data. Korean J Anesthesiol. 2013:64(5):402-6. https://doi.org/10.4097/kjae.2013.64.5.402

38. Mirowsky J. Reynolds Jr. age, depression, and attrition in the National Survey of families and households. Sociol Methods Res. 2000;28(4):476-504. https:// doi.org/10.1177/0049124100028004004.

39. Mack C, Su Z, Westreich D. Managing missing data in patient registries: addendum to registries for evaluating patient outcomes: a User's guide. 3rd ed. Rockville: AHRQ Methods for Effective Health Care; 2018.

40. Holmes J, House A. Psychiatric illness predicts poor outcome after surgery for hip fracture: a prospective cohort study. Psychol Med. 2000;30(4):921-9. https://doi.org/10.1017/S0033291799002548.

41. Sun T, Wang X, Liu Z, Chen $X$, Zhang J. Plasma concentrations of pro- and antiinflammatory cytokines and outcome prediction in elderly hip fracture patients. Injury. 2011;42(7):707-13. https://doi.org/10.1016/j.injury.2011.01.010.

42. Wiegertjes $R$, van de Loo FAJ, Blaney Davidson EN. A roadmap to target interleukin-6 in osteoarthritis. Rheumatology (Oxford). 2020;59(10):2681-94. https://doi.org/10.1093/rheumatology/keaa248.

43. Michaelides A, Zis P. Depression, anxiety and acute pain: links and management challenges. Postgrad Med. 2019;131(7):438-44. https://doi. org/10.1080/00325481.2019.1663705.

44. Bonasia DE, Palazzolo A, Cottino U, Saccia F, Mazzola C, Rosso F, et al. Modifiable and nonmodifiable predictive factors associated with the outcomes of Total knee arthroplasty. Joints. 2019;7(1):13-8. https://doi.org/1 0.1055/s-0039-1678563

45. Soysal P, Veronese N, Thompson T, Kahl KG, Fernandes BS, Prina AM, et al. Relationship between depression and frailty in older adults: a systematic review and meta-analysis. Ageing Res Rev. 2017;36:78-87. https://doi.org/1 0.1016/j.arr.2017.03.005.

46. Evans GW. The built environment and mental health. J Urban Health. 2003; 80(4):536-55. https://doi.org/10.1093/jurban/jtg063.

47. Liu RT, Kleiman EM, Nestor BA, Cheek SM. The hopelessness theory of depression: a quarter century in review. Clin Psychol (New York). 2015;22(4): 345-65. https://doi.org/10.1111/cpsp.12125.

48. WHO. International classification of diseases for mortality and morbidity statistics (11th Revision) 2021 [Available from: https://icd.who.int/browse11/ |-m/en]

49. Uzer A, Yucens B. Chronotype and depressive symptoms in healthy subjects: the mediating role of hopelessness and subjective sleep quality.
Chronobiol Int. 2020;37(8):1173-80. https://doi.org/10.1080/07420528.2020.1 775629.

50. Bond J, Gregson B, Smith M, Rousseau N, Lecouturier J, Rodgers $H$. Outcomes following acute hospital care for stroke or hip fracture: how useful is an assessment of anxiety or depression for older people? Int J Geriatr Psychiatry. 1998;13(9):601-10. https://doi.org/10.1002/(SICI)1099-11 66(199809)13:9<601::AID-GPS827>3.0.CO;2-B

51. Marin RS, Biedrzycki RC, Firinciogullari S. Reliability and validity of the apathy evaluation scale. Psychiatry Res. 1991;38(2):143-62. https://doi.org/10.1016/ 0165-1781(91)90040-V.

52. Renner $\mathrm{KH}$, Hock M, Bergner-Kother $\mathrm{R}$, Laux L. Differentiating anxiety and depression: the state-trait anxiety-depression inventory. Cogn Emot. 2018; 32(7):1409-23. https://doi.org/10.1080/02699931.2016.1266306.

53. Hayden JA, van der Windt DA, Cartwright $J \mathrm{~L}$, Cote $P$, Bombardier C. Assessing bias in studies of prognostic factors. Ann Intern Med. 2013;158(4): 280-6. https://doi.org/10.7326/0003-4819-158-4-201302190-00009.

54. Juni $P$, Witschi $A$, Bloch $R$, Egger $M$. The hazards of scoring the quality of clinical trials for meta-analysis. JAMA. 1999;282(11):1054-60. https://doi.org/1 $0.1001 /$ jama.282.11.1054

55. Morton SC, Murad MH, O'Connor E, Lee CS, Booth M, Vandermeer BW, et al. Quantitative synthesis-an update. Methods Guide for Effectiveness and Comparative Effectiveness Reviews. Rockville: AHRQ Methods for Effective Health Care; 2008

\section{Publisher's Note}

Springer Nature remains neutral with regard to jurisdictional claims in published maps and institutional affiliations.
Ready to submit your research? Choose BMC and benefit from:

- fast, convenient online submission

- thorough peer review by experienced researchers in your field

- rapid publication on acceptance

- support for research data, including large and complex data types

- gold Open Access which fosters wider collaboration and increased citations

- maximum visibility for your research: over $100 \mathrm{M}$ website views per year

At $\mathrm{BMC}$, research is always in progress.

Learn more biomedcentral.com/submissions 